\title{
Alterations in the expression of Hs1-associated protein X-1 in the rat retina after optic nerve crush
}

\author{
LING CUI*, WEN-JING HE* , FAN XU*, LI JIANG, MING-LIANG LV, HUI HUANG, \\ JI-PING XU, YU WU, HAI-BIN ZHONG, SHAO-YANG ZHANG, LI-FEI CHEN, \\ CHAO-LAN SHEN, GANG YAO, LI LI, MIN LI and SI-MING ZENG \\ Department of Ophthalmology, People's Hospital of Guangxi Zhuang Autonomous Region, \\ Nanning, Guangxi 530021, P.R. China
}

Received September 1, 2015; Accepted September 7, 2016

DOI: $10.3892 / \mathrm{mmr} .2016 .5824$

\begin{abstract}
HS-1-associated protein X-1 (Hax-1) has been suggested to be expressed in various rodent and human tissues. Accumulating evidence has demonstrated that Hax-1 exerts an anti-apoptotic effect in neurological diseases. Furthermore, it has also been reported that Hax-1 interacts with various apoptosis-associated proteins, including high temperature-regulated A2 (HtrA2) and caspase-3. Previous studies have indicated that abnormal expression of Hax-1 may be associated with the development of the nervous system and with the pathophysiology of neurological diseases, including traumatic brain injury and cerebral ischemia. The present study reported temporal-spatial patterns of Hax-1 in rat retina following optic nerve crush (ONC). Using western blotting and double-immunofluorescence, significant upregulation of Hax-1 was observed in retinal ganglion cells (RGCs) in the retina following ONC. Increased Hax-1 expression was demonstrated to be accompanied by upregulation of active-caspase-3 and $\mathrm{HtrA} 2$ following ONC. In addition, Hax-1 co-localized with active caspase- 3 and HtrA2 in RGCs following ONC. Terminal deoxynucleotidyl transferase-mediated biotinylated-dUTP nick-end labeling staining suggested that Hax-1 was involved in RGC apoptosis following ONC. Thus, these results suggested that Hax-1 may participate
\end{abstract}

Correspondence to: Dr Si-Ming Zeng or Dr Min Li, Department of Ophthalmology, People's Hospital of Guangxi Zhuang Autonomous Region, 6 Taoyuan Road, Nanning, Guangxi 530021, P.R. China

E-mail: eyezsm@163.com

E-mail: eyeminli@163.com

Abbreviations: Hax-1, HS-1-associated protein X-1; HtrA2, high-temperature-regulated A2; ONC, optic nerve crush; RGCs, retinal ganglion cells; PAGE, polyacrylamide gel electrophoresis; TUNEL, terminal deoxynucleotidyl transferase-mediated biotinylated-dUTP nick-end labeling; SEM, standard error of mean

*Contributed equally

Key words: HS-1-associated protein X-1, optic nerve crush, retinal ganglion cells, apoptosis, rat in regulating RGC apoptosis via interacting with caspase-3 and HtrA2 following ONC.

\section{Introduction}

Numerous studies have demonstrated that the majority of retinal ganglion cells (RGCs) undergo cell death following optic nerve (ON) injury, which leads to irreversible visual impairment (1-4). The ON crush (ONC) model is commonly used to analyze neurodegenerative processes in the optic nerve and retina (2-4). It is well known that delayed RGC apoptosis is activated following ONC. However, the detailed mechanisms underlying RGC apoptosis following ONC remain to be elucidated.

HS-1-associated protein X-1 (Hax-1) was identified as a $35 \mathrm{kDa}$ multi-functional protein encoded by the Hax-1 gene (5), and was suggested to be expressed in various rodent and human tissues (6-8). Previous studies have demonstrated that abnormal expression of Hax-1 may be associated with development and disease of the nervous system, including traumatic brain injury and cerebral ischemia (9-11). It has also been reported that Hax-1 interacts with various apoptosis-associated proteins, including high temperature regulated A2 (HtrA2) and caspase-3 (7). Thus, it is possible that Hax-1 is important in RGC apoptosis following ONC. However, its regulatory mechanism following ON injury remains unknown.

The present study demonstrated temporal-spatial patterns of Hax-1 expression in rat retina following ONC. This research may improve understanding of the physiological functions of Hax-1 in apoptosis of RGCs following ONC, and its association with the cellular and molecular mechanisms.

\section{Materials and methods}

Experimental animals. A total of 96 rats (age, 6-8 weeks) were handled in accordance with the Association for Research in Vision and Ophthalmology Statement on Use of Animals in Ophthalmic and Vision Research, and experimental protocols were approved by the Ethics Committee of the People's Hospital of Guangxi Zhuang Autonomous Region (Nanning, China). Healthy male Sprague-Dawley rats (weight, 220-275 g) supplied by the Medical Laboratory Animal Center (Guangxi Medical University, Guangxi, China) were kept under standardized 
conditions with a $12 \mathrm{~h}$ light/dark cycle, temperature of $\sim 23^{\circ} \mathrm{C}$ and $60 \%$ humidity. They had access to rodent chow and water was available. The animals were used for western blotting analysis ( 8 groups of $8, n=64$ ), immunofluorescence studies ( 2 groups of $8, \mathrm{n}=16$ ) and terminal deoxynucleotidyl transferase-mediated biotinylated-dUTP nick-end labeling (TUNEL) staining (2 groups of $8, n=16)$.

Surgery. Briefly, rats were anesthetized with $7 \%$ chloral hydrate solution ( $6 \mathrm{ml} / \mathrm{kg}$ body weight) and the surgical procedure to produce the ONC model was performed as described previously (3). The left ON was exposed under a surgical microscope and crushed with forceps $2 \mathrm{~mm}$ behind the eye for $10 \mathrm{sec}$. A sham operation was performed on the right $\mathrm{ON}$, in which the ON was exposed but not crushed.

Tissue lysis and western blotting. The rats were sacrificed at different time points by administering an overdose of anesthesia (650 mg/kg chloral hydrate). Retina tissues were harvested and stored at $-80^{\circ} \mathrm{C}$ until use. Cells were lysed in lysis buffer to collect total protein $[50 \mathrm{mmol} / \mathrm{l}$ Tris $(\mathrm{pH} 7.5), 1 \%$ Triton X-100, $1 \%$ NP-40, $10 \%$ sodium dodecyl sulfate, $0.5 \%$ sodium deoxycholate, $5 \mathrm{mmol} / \mathrm{l}$ Tris EDTA, $10 \mu \mathrm{g} / \mathrm{ml}$ leupeptin and $10 \mu \mathrm{g} / \mathrm{ml}$ aprotinin) and centrifuging for $20 \mathrm{~min}$ at $15,000 \times \mathrm{g}$ in a microcentrifuge at $4^{\circ} \mathrm{C}$ was conducted to collect the supernatant. Protein concentrations were determined using the Bradford assay (Bio-Rad Laboratories, Inc., Hercules, CA, USA). The resulting supernatants (50 $\mu \mathrm{g}$ protein) were subsequently subjected to $10 \%$ SDS-polyacrylamide gel electrophoresis and transferred to polyvinylidine difluoride membranes (EMD Millipore, Billerica, MA, USA). Following a blocking step with 5\% non-fat milk, primary antibodies against Hax-1 (1:500; cat. no. sc-28268; Santa Cruz Biotechnology, Inc., Dallas, TX, USA), active caspase-3 (1:1,000; cat. no. 9661; Cell Signaling Technology, Inc., Danvers, MA, USA), HtrA2 (1:500; cat. no. sc-25606; Santa Cruz Biotechnology, Inc.) and $\beta$-actin (1:1,000; cat. no. ab8227; Abcam, Cambridge, MA, USA) were incubated with the membrane at $4^{\circ} \mathrm{C}$ overnight. Subsequently, the membranes were washed in Tris/ $\mathrm{HCl}$ saline buffer supplemented with $0.1 \%$ Tween-20 (pH 7.4) three times at room temperature (5 $\mathrm{min} /$ wash). Following incubation with the appropriate horseradish peroxidase-conjugated goat anti-rabbit secondary antibody (1:2,000; cat. no. 4030-05; SouthernBiotech, Birmingham, AL, USA) for $2 \mathrm{~h}$ at room temperature, the blots were developed using an enhanced chemiluminescence system (Pierce; Thermo Fisher Scientific, Inc., Waltham, MA, USA).

Immunofluorescence. The rats ( $\mathrm{n}=3$ per time point) were anesthetized at the designated time points and perfused with saline $(20 \mathrm{ml})$ followed by $4 \%$ paraformaldehyde and $0.5 \%$ picric acid in $0.1 \mathrm{M}$ phosphate buffer ( $\mathrm{pH} 7.4,20 \mathrm{ml})$. Subsequently, the rats were fixed in $4 \%$ paraformaldehyde overnight at $4^{\circ} \mathrm{C}$. The eyes were dehydrated and cryosections $(7 \mu \mathrm{m})$ were produced. Briefly, the cryosections were washed with $1 \mathrm{X}$ Tris-buffered saline twice, and then were blocked with $1 \%$ bovine serum albumin (Wuhan Boster Biological Technology Ltd., Wuhan, China) for $1 \mathrm{~h}$. Subsequently, the sections were incubated with antibodies against anti-Hax-1 (1:200; cat. no. sc-34273; Santa Cruz Biotechnology, Inc.), and neuronal marker of RGCs, anti-NeuN (1:500; cat. no. ABN78; EMD Millipore), anti-HtrA2
A

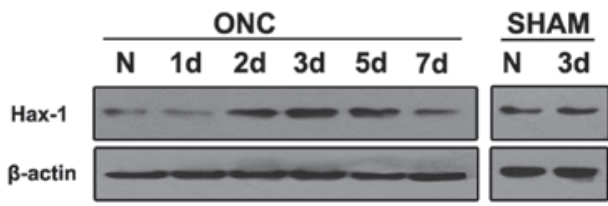

B

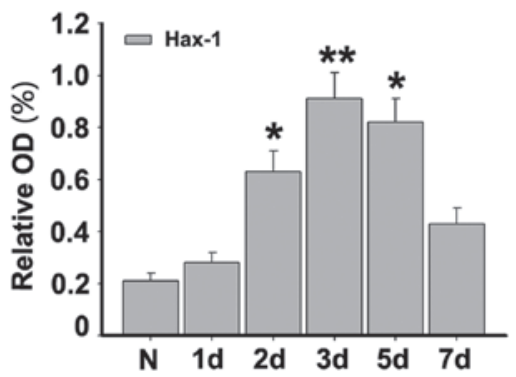

Figure 1. Temporal protein expression of Hax-1 analyzed by western blotting following ONC. (A) Western blotting was used to quantify the temporal patterns of Hax-1 expression in the retina, which were prepared at the designated time points following ONC. (B) Bar chart to indicate the ratio of Hax-1 to $\beta$-actin at each time point. $\beta$-actin was used to confirm equal loading of proteins on the gel. The data are presented as the mean \pm standard error of the mean $(\mathrm{n}=3)$. ${ }^{*} \mathrm{P}<0.05,{ }^{* *} \mathrm{P}<0.01$ vs. the $\mathrm{N}$ group. Hax-1, Hs1-associated protein $\mathrm{X}-1$; ONC, optic nerve crush; OD, optical density; N, normal.

(1:200; cat. no. sc-25606; Santa Cruz Biotechnology, Inc.) and apoptosis marker anti-active caspase-3 (1:200; cat. no. sc-22171; Santa Cruz Biotechnology, Inc.) overnight at $4^{\circ} \mathrm{C}$ in a humidified box. Following incubation with the primary antibodies, the cryosections were washed three times in phosphate-buffered saline (PBS), followed by an incubation with donkey anti-goat Alexa Fluor ${ }^{\circledR} 488$ (cat. no. ab150129) and donkey anti-rabbit Alexa Fluor $^{\circledR} 555$ (cat. no. ab150074) secondary antibodies (1:1,000; Abcam) for $2 \mathrm{~h}$ at $4^{\circ} \mathrm{C}$. The stained sections were examined at x20 or x40 magnification on a fluorescence microscope (Leica Microsystems GmbH, Wetzlar, Germany).

TUNEL staining. TUNEL staining was employed using the In Situ Cell Death Detection Kit, Fluorescence (Roche Applied Science, Mannheim, Germany). The cryosections were rinsed with PBS and treated with 1\% Triton-100 in PBS for 2 min on ice. The Slides were rinsed in PBS and incubated for $60 \mathrm{~min}$ at $37^{\circ} \mathrm{C}$ with $50 \mu \mathrm{l}$ of TUNEL reaction mixture. Following washing with PBS three times, the slides were analyzed on a fluorescence microscope.

Quantitative analysis. A total of three adjacent sections (50 $\mu \mathrm{m}$ apart) per animal were obtained for each rat. Sections were double labeled for Hax-1 with phenotype-specific markers, NeuN and active caspase-3. The number of TUNEL and Hax-1/TUNEL-positive cells in the ganglion cell layer (GCL) was counted in a 400x400 $\mu$ m measuring frame. The cell counts in the three or four adjacent sections were then used to determine the total number of TUNEL-positive cells or Hax-1/TUNEL-positive cells $/ \mathrm{mm}^{2}$. A minimum of 200 TUNEL-positive cells were counted in each section.

Statistical analysis. All collected data were analyzed with SPSS software (version 13.0; SPSS, Inc., Chicago, IL, USA). Data are presented as the mean \pm standard error of the mean and each experiment was repeated at least three times. All statistical 

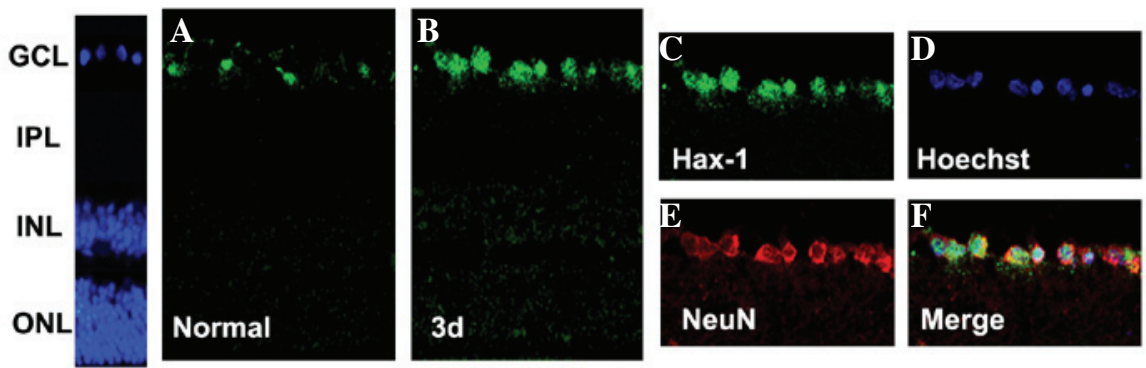

Figure 2. Changes in expression and distribution of Hax-1 visualized by immunofluorescence staining in the retina following ONC. (A) The normal retina demonstrated weak immunoreactivity for Hax-1 antibody. (B) At 3 days after ONC, the number of positive cells significantly increased in the GCL. (C-F) At 3 days after ONC, increased Hax-1 co-stained with RGCs in the GCL. (C) Hax-1 (green), (D) nuclear Hoechst staining (blue) and (E) NeuN (red). (F) The yellow color visualized in the merged images represented the co-localization of Hax-1 with NeuN. Scale bars are equivalent to $60 \mu \mathrm{m}$. Hax-1, Hs1-associated protein X-1; ONC, optic nerve crush; GCL, ganglion cell layer; RGCs, retinal ganglion cells.

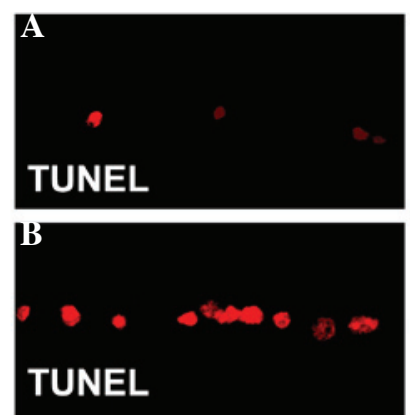

$\mathbf{F}$
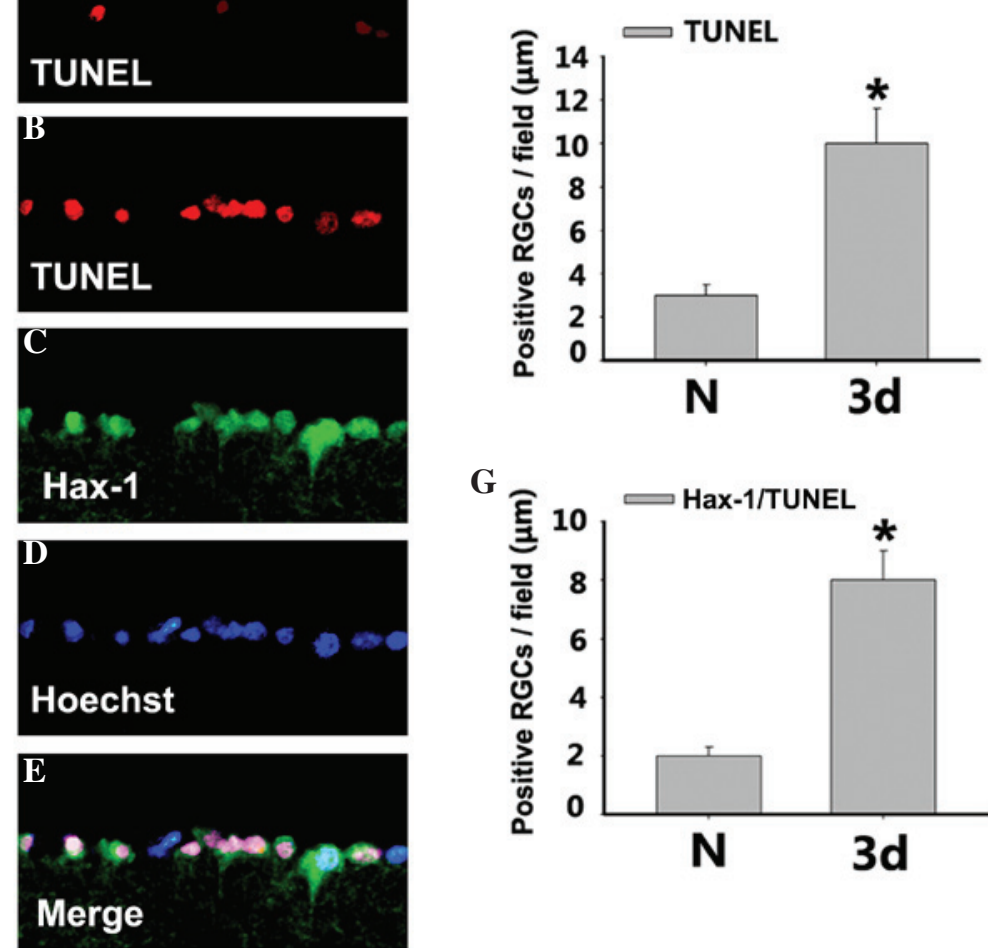

G

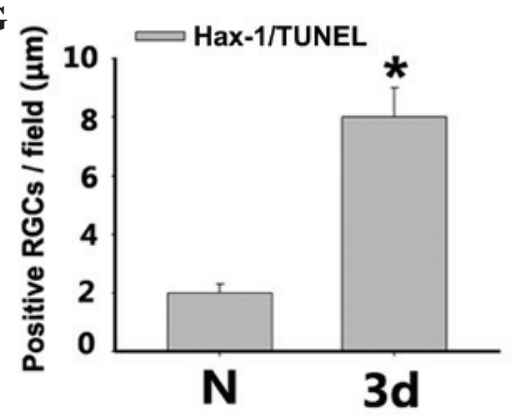

Figure 3. Expression and location of TUNEL-positive cells and Hax-1 in the adult rat retina following ONC. (A) TUNEL staining indicating the expression of TUNEL-positive cells (red) was weak in the sham group. (B) TUNEL-positive cells (red) were observed to be increased 3 days after ONC, (C) Hax-1 (green), (D) nuclear Hoechst staining (blue). (E) Yellow in the merged images represents the co-localization of Hax-1 with TUNEL-positive cells in the GCL. Scale bars are equivalent to $100 \mu \mathrm{m}$. (F and G) Semi-quantitative analysis indicated a significant increase in the density of TUNEL and Hax-1/TUNEL-positive RGCs in the GCL at 3 days after ONC. The data are presented as the mean \pm standard error of the mean $(n=3){ }^{*} \mathrm{P}<0.05$ vs. the $\mathrm{N}$ group. Hax-1, Hs1-associated protein X-1; ONC, optic nerve crush; GCL, ganglion cell layer; RGCs, retinal ganglion cells; N, normal; TUNEL, terminal deoxynucleotidyl transferase-mediated biotinylated-dUTP nick-end labeling.

analyses were determined by one-way analysis of variance followed by Tukey's post-hoc multiple comparison tests. $\mathrm{P}<0.05$ was considered to indicate a statistically significant difference.

\section{Results}

Temporal Hax-1 protein expression by western blotting following $O N C$. To quantify the expression pattern of Hax-1 in the retina, western blotting was conducted at the designated time points following ONC. It was demonstrated that Hax-1 was low in sham-operated retina tissue and maintained that level at 1 day after ONC. Subsequently, Hax-1 protein expression gradually increased and reached a maximal value at 3 days $(\mathrm{P}<0.01)$, prior to returning to normal levels on day 7 (Fig. 1). These results suggested that Hax-1 protein may be upregulated by ON injury.

Changes in expression and distribution of Hax-1 indicated by immunofluorescence staining in the retina following ONC. 
A
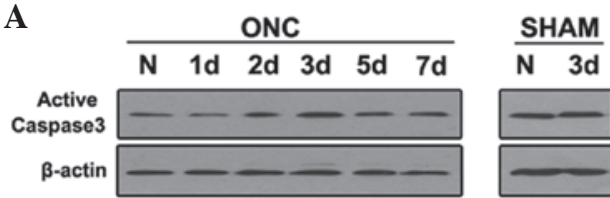

B

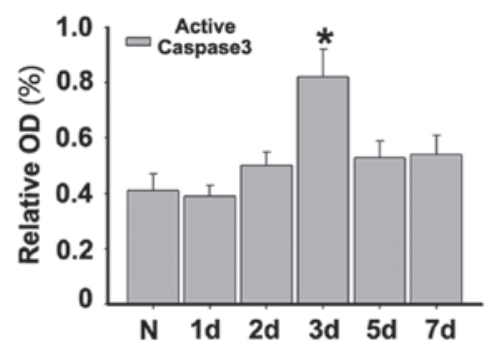

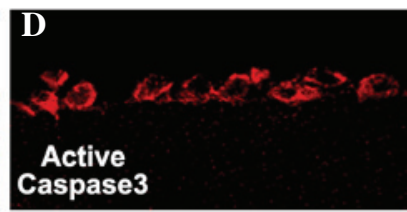

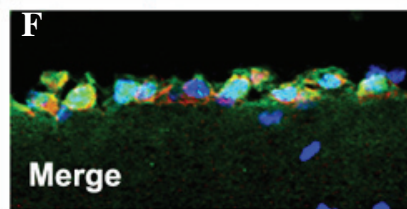

Figure 4. Hax-1 was associated with RGC apoptosis in retinas following ONC. (A) Western blotting demonstrated expression levels of active-caspase-3 in retinas following ONC. The expression of active caspase- 3 was increased following ONC and peaked at 3 days. (B) Bar chart demonstrates the ratio of active-caspase- 3 to $\beta$-actin at each time point. The data are presented as the mean \pm standard error of the mean $(n=3){ }^{*} \mathrm{P}<0.05$ vs. the $\mathrm{N}$ group. (C-F) Double immunofluorescence staining for Hax-1 immunoreactivity and active caspase-3 in the retina following ONC. (C) Hax-1 (green) activity colocalized with (D) active caspase-3 (red) and (E) nuclear Hoechst staining (blue) in (F) the merged image (yellow). Scale bars are equivalent to $30 \mu \mathrm{m}$. Hax-1, Hs1-associated protein X-1; ONC, optic nerve crush; OD, optical density; $\mathrm{N}$, normal.

To investigate the distribution of Hax-1 following ONC, double-labeling immunocytochemistry was used to determine the cellular localization of Hax-1 and NeuN (a marker of RGCs; Fig. 2). As Hax-1 protein reached a maximal value at 3 days after ONC, this time point was selected for further examination. Hax-1 expression was demonstrated in RGCs. Notably, the expression was markedly increased in RGCs at 3 days after ONC compared with the sham retina (Figs. 2A and B). Hax-1 expression was predominantly located in NeuN-positive cells (Fig. 2C-F). These findings indicated that the temporal pattern of Hax-1 following ONC was consistent with the results of the western blotting, and suggested the localization of Hax-1 appeared to be confined to the RGCs.

Hax-1 was relevant to $R G C$ apoptosis in retinal tissue following ONC. In order to investigate the apoptosis of RGCs, TUNEL staining was performed at 3 days after ONC to further determine whether Hax-1 expression was involved in the changes in RGC biological functions, including apoptosis (Fig. 3). Apoptosis of RGCs, as assessed by TUNEL staining, was relatively lower in retinas from the sham-operated group

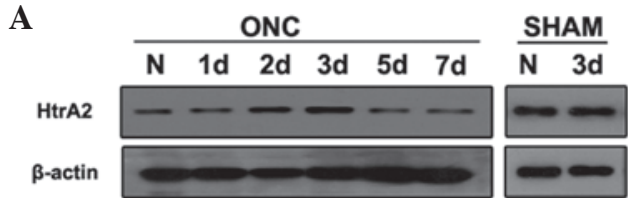

B
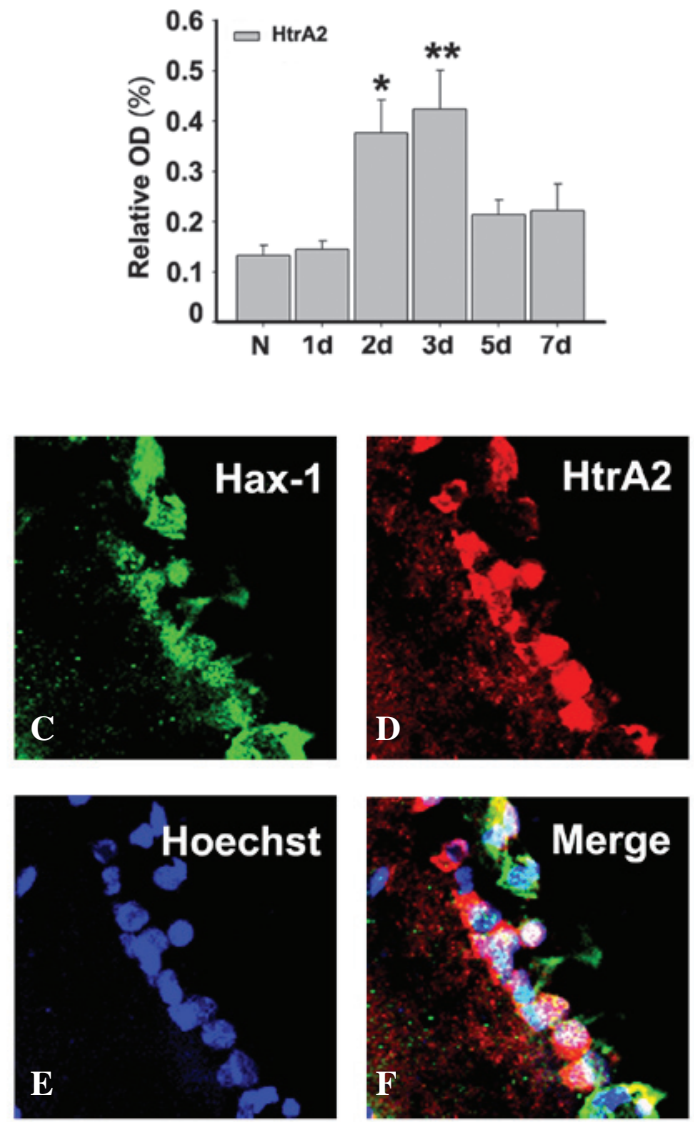

Figure 5. Association of Hax-1 with HtrA2 in the retina following ONC. (A) Western blotting demonstrated expression levels of HtrA2 in the retina following ONC. The expression of HtrA2 was increased following ONC and peaked at 3 days. (B) Bar chart demonstrates the ratio of $\mathrm{HtrA} 2$ to $\beta$-actin at each time point. The data are presented as the mean \pm standard error of the mean $(\mathrm{n}=3){ }^{*} \mathrm{P}<0.05,{ }^{* *} \mathrm{P}<0.01$ vs. the normal group. (C-F) Double immunofluorescence staining for Hax-1 immunoreactivity (green) and HtrA2 (red) in the retina following ONC. (C) Hax-1 (green) activity colocalized with (D) HtrA2 (red) and (E) nuclear Hoechst staining (blue) in (F) the merged image (yellow). Scale bars are equivalent to $30 \mu \mathrm{m}$. Hax-1, Hs1-associated protein X-1; HtrA2, high temperature-regulated A2; ONC, optic nerve crush; OD, optical density; N, normal.

(Fig. 3A). The number of TUNEL and Hax-1/TUNEL-positive cells was significantly increased at 3 days after ONC (Fig. 3B-G; P<0.05).

Caspase-3 represents a specific marker of a subpopulation of apoptotic cells. Western blots were used to determine the expression levels of active caspase-3. It was demonstrated that the expression of active caspase-3 was relatively low in normal retinas, but it increased and reached a maximum at 3 days after ONC (Figs. 4A and $\mathrm{B} ; \mathrm{P}<0.05$ ). To further investigate the distribution and co-localization of Hax-1 and active caspase-3 at 3 days after ONC, double immunofluorescent microscopy was performed to detect active caspase- 3 and Hax-1. The results demonstrated that the co-localization of Hax-1 and active-caspase- 3 was observed at 3 days after ONC 
(Fig. 4C-F). These results indicated that Hax-1 is important in RGC apoptosis in a caspase-dependent way following ONC.

Association of Hax-1 with HtrA2 in the retina following ONC. To investigate the exact role of Hax-1 in RGC apoptosis following $\mathrm{ONC}$, western blotting was performed to determine the expression levels of HtrA2. HtrA2 exhibited a marked upregulation at 2 and 3 days after $\mathrm{ONC}$ (Figs. 5A and $\mathrm{B} ; \mathrm{P}<0.05$ and $\mathrm{P}<0.01$, respectively). Furthermore, the co-localization of Hax-1 and HtrA2 was observed by double immunofluorescent stain at 3 days after ONC (Fig. 5C-F).

\section{Discussion}

ONC initiates a host of progressive molecular and cellular events, which evolve over the subsequent hours and days, resulting in neuronal survival, apoptosis, reactive gliosis and inflammation (12-15). These secondary events lead to additional cell apoptosis, axonal degeneration, tissue injury, impaired regeneration, and functional disabilities $(1,16,17)$. RGC apoptosis is one of the most severe responses following ON injury, it may result in loss of RGCs and poor visual function (18-20). Thus, improved understanding of the molecular mechanisms involved in the death of RGCs would benefit the development of neuroprotective therapeutic strategies for the treatment of $\mathrm{ON}$ injury. However, the exact mechanism underlying RGC death following ON injury remains to be elucidated.

In the present study, the expression profiles of Hax-1 in adult rat retina were investigated following acute $\mathrm{ON}$ injury. It was demonstrated that the protein expression levels of Hax-1 were significantly upregulated following ONC. Furthermore, it was observed that Hax-1 was significantly increased in the GCL at 3 day after ONC, as indicated by immunofluorescent staining. In addition, based on the double immunofluorescent staining, the co-localization of Hax-1 and NeuN was detected in the retina. This result suggested that the localization of Hax-1 appeared to be confined to the RGCs. The co-localization of Hax-1/active caspase-3 and Hax-1/TUNEL-positive cells was detected in RGCs, thus suggesting that Hax-1 may participate in RGC apoptosis regulation. Quantitative analysis indicated that the expression patterns of active caspase-3 and TUNEL-positive cells were parallel with that of Hax-1. These data were consistent with the hypothesis that Hax-1 was implicated in pathophysiology of ONs following ONC.

Previous studies have demonstrated that Hax-1 is important in nervous system disorders, including traumatic brain injury, cerebral ischemia and seizure-induced hippocampal injury (9-11). It has been widely reported that Hax-1 interacts with apoptosis-associated proteins, including HtrA2, protease-activated receptor 1 (Par1), caspase-3, caspase-9 or Bcl-2-associated $\mathrm{X}$ protein $(8,21-23)$. Numerous studies have demonstrated that the activation of caspase- 3 was the key effector of RGC apoptosis following ONC (24-26). Previously, Hax-1 has been identified as a new substrate of caspase-3, which prevents apoptosis by inhibiting the catalytic activation of caspase-3 (27). Caspase-3 cleaves Hax-1 at the Asp127 residue during apoptosis (27). Conversely, overexpression of Hax-1 had an inhibitory effect on caspase-3 activity $(22,27,28)$. In the present study, the temporal pattern of the expression of Hax-1 is paralleled with the expression of active caspase- 3 in the retina following ONC. As Hax-1 has these antiapoptotic features, it was hypothesized that upregulation of Hax-1 may inhibit the caspase-3-mediated process of RGC apoptosis following ONC.

In addition, Hax-1 has been demonstrated to interact with HtrA2 via Parl and to promote survival of lymphocytes and neurons $(23,29)$. HtrA2 has been identified as an apoptosis-regulating protein (30-32). During apoptosis, HtrA2 is released from the intermembrane space of the mitochondria into the cytosol, where it likely promotes neuronal cell death via promoting caspase activation. In the current study, HtrA2 expression was paralleled with that of Hax-1 in the retina following ONC. These results suggested that Hax-1 may participate in regulating RGC apoptosis via interacting with caspase-3 and HtrA2 following ONC.

In conclusion, the present study is the first, to the best of our knowledge, to demonstrate that Hax-1 is significantly upregulated in RGCs after ONC. These findings suggested that Hax-1 may participate in regulating RGC apoptosis after ONC. Further studies are required to confirm whether Hax-1 has neuroprotective functions following $\mathrm{ONC}$, which may aid understanding of a novel molecular pathway of RGC apoptosis following ONC, and a novel strategy for the treatment of $\mathrm{ON}$ injury.

\section{Acknowledgements}

The present study was supported by the National Natural Science Foundation of China (grant nos. 81460087, 81560166, 81660161 and 81660168), the Natural Science Foundation of Guangxi Zhuang Autonomous Region (grant nos. 2012GXNSFAA276039 and 2011GXNSFA018228) and the Science Fund Project of People's Hospital of Guangxi Zhuang Autonomous Region (grant nos. qn2014-1 and qn2014-2).

\section{References}

1. Li HY, Ruan YW, Ren CR, Cui Q and So KF: Mechanisms of secondary degeneration after partial optic nerve transection. Neural Regen Res 9: 565-574, 2014.

2. Schwartz M: Optic nerve crush: Protection and regeneration. Brain Res Bull 62: 467-471, 2004.

3. Xu F, Huang H, Wu Y, Lu L, Jiang L, Chen L, Zeng S, Li L and $\mathrm{Li} \mathrm{M}$ : Upregulation of Gem relates to retinal ganglion cells apoptosis after optic nerve crush in adult rats. J Mol Histol 45: 565-571, 2014.

4. Wu Y, Xu F, Huang H, Chen L, Wen M, Jiang L, Lu L, Li L, Song D, Zeng S, et al: Up-regulation of SKIP relates to retinal ganglion cells apoptosis after optic nerve crush in vivo. J Mol Histol 45: 715-721, 2014.

5. Suzuki Y, Demoliere C, Kitamura D, Takeshita H, Deuschle U and Watanabe T: HAX-1, a novel intracellular protein, localized on mitochondria, directly associates with HS1, a substrate of Src family tyrosine kinases. J Immunol 158: 2736-2744, 1997.

6. Simmen T: Hax-1: A regulator of calcium signaling and apoptosis progression with multiple roles in human disease. Expert Opin Ther Targets 15: 741-751, 2011.

7. Yap SV, Koontz JM and Kontrogianni-Konstantopoulos A: HAX-1: A family of apoptotic regulators in health and disease. J Cell Physiol 226: 2752-2761, 2011.

8. Fadeel B and Grzybowska E: HAX-1: A multifunctional protein with emerging roles in human disease. Biochim Biophys Acta 1790: 1139-1148, 2009.

9. $\mathrm{Hu} \mathrm{J}, \mathrm{Mu} \mathrm{C}$ and Hao J: Cerebral ischemia reduces expression of Hs1-associated protein X-1 (Hax-1) in mouse brain. Neurosci Lett 534: 338-343, 2013.

10. Rami A, Kim M, Niquet $\mathrm{J}$ and Langhagen A: Alterations in the expression of the anti-apoptotic factor HAX-1 upon seizures-induced hippocampal injury in the neonatal rat brain. Neurochem Res 37: 116-125, 2012. 
11. Shi W, Zhao W, Shen A, Shao B, Wu X, Yang J, Ni L, Wu Q and Chen J: Traumatic brain injury induces an up-regulation of Hs1-associated protein X-1 (Hax-1) in rat brain cortex. Neurochem Res 36: 375-382, 2011.

12. Suzuki H, Oku H, Horie T, Morishita S, Tonari M, Oku K, Okubo A, Kida T, Mimura M, Fukumoto M, et al: Changes in expression of aquaporin-4 and aquaporin-9 in optic nerve after crushing in rats. PLoS One 9: e114694, 2014.

13. Mac Nair CE, Fernandes KA, Schlamp CL, Libby RT and Nickells RW: Tumor necrosis factor alpha has an early protective effect on retinal ganglion cells after optic nerve crush. J Neuroinflammation 11: 194, 2014.

14. Yang L, Miao L, Liang F, Huang H, Teng X, Li S, Nuriddinov J, Selzer ME and $\mathrm{Hu}$ Y: The mTORC1 effectors S6K1 and 4E-BP play different roles in CNS axon regeneration. Nat Commun 5: $5416,2014$.

15. Huang SP and Tsai RK: Efficacy of granulocyte-colony stimulating factor treatment in a rat model of anterior ischemic optic neuropathy. Neural Regen Res 9: 1502-1505, 2014.

16. Morgan-Warren PJ, Berry M, Ahmed Z, Scott RA and Logan A: Exploiting mTOR signaling: A novel translatable treatment strategy for traumatic optic neuropathy? Invest Ophthalmol Vis Sci 54: 6903-6916, 2013.

17. Watanabe M: Regeneration of optic nerve fibers of adult mammals. Dev Growth Differ 52: 567-576, 2010.

18. Schmitt HM, Pelzel HR, Schlamp CL and Nickells RW: Histone deacetylase 3 (HDAC3) plays an important role in retinal ganglion cell death after acute optic nerve injury. Mol Neurodegener 9: 39, 2014.

19. Shibeeb O, Wood JP, Casson RJ and Chidlow G: Effects of a conventional photocoagulator and a 3-ns pulse laser on preconditioning responses and retinal ganglion cell survival after optic nerve crush. Exp Eye Res 127: 77-90, 2014.

20. Chen M,Xiang Z and Cai J: The anti-apoptotic and neuro-protective effects of human umbilical cord blood mesenchymal stem cells (hUCB-MSCs) on acute optic nerve injury is transient. Brain Res 1532: 63-75, 2013.

21. Chao JR, Parganas E, Boyd K, Hong CY, Opferman JT and Ihle JN: Hax1-mediated processing of HtrA2 by Parl allows survival of lymphocytes and neurons. Nature 452: 98-102, 2008.
22. Han Y, Chen YS, Liu Z, Bodyak N, Rigor D, Bisping E, Pu WT and Kang PM: Overexpression of HAX-1 protects cardiac myocytes from apoptosis through caspase-9 inhibition. Circ Res 99: 415-423, 2006.

23. Cilenti L, Soundarapandian MM, Kyriazis GA, Stratico V, Singh S, Gupta S, Bonventre JV, Alnemri ES and Zervos AS: Regulation of HAX-1 anti-apoptotic protein by Omi/HtrA2 protease during cell death. J Biol Chem 279: 50295-50301, 2004.

24. Wang Y, Zhang H, Liu Y, Li P, Cao Z and Cao Y: Erythropoietin (EPO) protects against high glucose-induced apoptosis in retinal ganglional cells. Cell Biochem Biophys 71: 749-755, 2015.

25. Cao Y, Li X, Shi P, Wang LX and Sui ZG: Effects of L-carnitine on high glucose-induced oxidative stress in retinal ganglion cells. Pharmacology 94: 123-130, 2014.

26. Rathnasamy G, Sivakumar V, Rangarajan P, Foulds WS, Ling EA and Kaur C: NF-kB-mediated nitric oxide production and activation of caspase- 3 cause retinal ganglion cell death in the hypoxic neonatal retina. Invest Ophthalmol Vis Sci 55: 5878-5889, 2014

27. Lee AY, Lee Y, Park YK, Bae KH, Cho S, Lee DH, Park BC, Kang S and Park SG: HS 1-associated protein X-1 is cleaved by caspase-3 during apoptosis. Mol Cells 25: 86-90, 2008.

28. Yedavalli VS, Shih HM, Chiang YP, Lu CY, Chang LY, Chen MY, Chuang CY, Dayton AI, Jeang KT and Huang LM: Human immunodeficiency virus type $1 \mathrm{Vpr}$ interacts with antiapoptotic mitochondrial protein HAX-1. J Virol 79: 13735-13746, 2005.

29. Li B, Hu Q, Wang H, Man N, Ren H, Wen L, Nukina N, Fei E and Wang G: Omi/HtrA2 is a positive regulator of autophagy that facilitates the degradation of mutant proteins involved in neurodegenerative diseases. Cell Death Differ 17: 1773-1784, 2010.

30. Zurawa-Janicka D, Skorko-Glonek J and Lipinska B: HtrA proteins as targets in therapy of cancer and other diseases. Expert Opin Ther Targets 14: 665-679, 2010.

31. Bhuiyan MS and Fukunaga K: Mitochondrial serine protease HtrA2/Omi as a potential therapeutic target. Curr Drug Targets 10: 372-383, 2009.

32. Sun H, Li L, Zhou F, Zhu L, Ke K, Tan X, Xu W, Rui Y, Zheng H, Zhou Z and Yang H: The member of high temperature requirement family HtrA2 participates in neuronal apoptosis after intracerebral hemorrhage in adult rats. J Mol Histol 44: 369-379, 2013. 